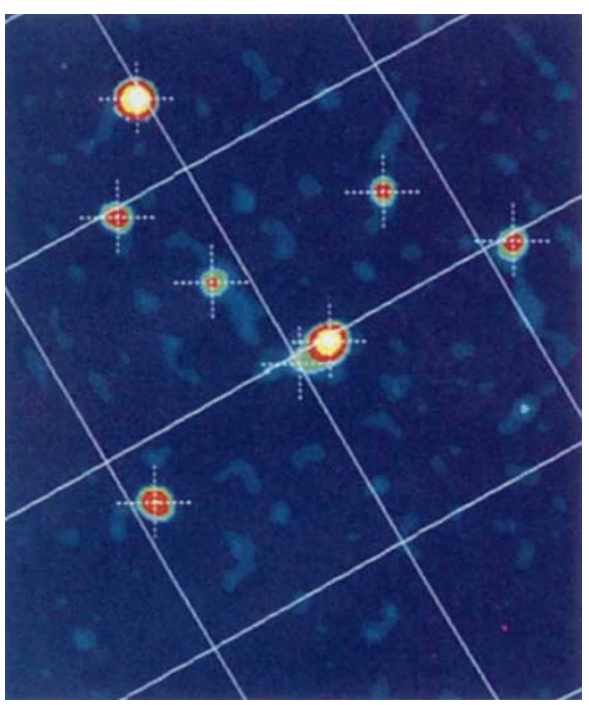

Close, but not close enough - X-ray sources ${ }^{1}$ near the heart of our Galaxy (gridlines are at $5^{\circ}$ intervals).

tational energy, was and is the simpler hypothesis.

The idea seemed to be confirmed most dramatically this May in images of the nucleus of the nearby active radio galaxy, M87, from the restored Hubble Space Telescope ${ }^{7}$. A central gaseous disk could be seen, and the measured gas velocities (again, just proportional to $(G M / R)^{0.5}$ ) convincingly required the central mass of the nucleus to be about $2 \times 10^{9} M_{\odot}$, far in excess of the visible matter and stars but about what had been suspected for a central black hole. Similar measurements of the gas clouds near our own Galactic Centre $^{8}$ had long suggested a central (unseen) mass of some $10^{6} M_{\odot}$.

So the lack of detectable hard X-ray emission from Sagittarius $A^{*}$, the supposed Galactic Centre, strains an already growing problem: why is there no sign of accretion onto the central black hole from the gas clouds (and possibly mass-losing stars in a dense central cluster) observed in the central few parsecs? The long history of X-ray detection of sources coincident with or near Sgr A* had always pointed to a luminosity problem ${ }^{8}$, as the measured soft X-ray luminosity was no more than about $10^{35} \mathrm{erg} \mathrm{s}^{-1}$, or only about $10^{-7}$ of the Eddington luminosity (if the spectrum were extrapolated to $\sim 100$ $\mathrm{keV}$ ) for a $10^{6} M_{\odot}$ black hole. But more luminous hard X-ray or soft gamma-ray sources had been seen somewhere near the Galactic Centre with earlier nonimaging detectors and so it was hoped that Sgr $A^{*}$ was a respectable active galactic nucleus after all.

The new observations from the Sigma/ GRANAT telescope ${ }^{1}$, with unprecedented total exposure time of nearly 1,800 hours over 4 years, show that this is not the case. The luminous hard X-ray sources around the Galactic Centre are in fact isolated, discrete black-hole candi- dates, accreting matter either from binary companions (and one X-ray nova is included) or from dense molecular clouds through which they are drifting. The newly developed coded aperture technique for hard X-ray imaging had already revealed $^{9}$, in earlier balloon-borne hard $\mathrm{X}$-ray images, at least one transient blackhole candidate in the $\mathrm{Sgr} \mathrm{A}^{*}$ region, with possible backscattered emission at 511 $\mathrm{keV}$ (this is an indication of positron annihilation, which was also probably directly detected by Sigma from a flare of the brightest black-hole candidate ${ }^{1}$ ). The Galactic Centre region alone suggests that the whole Galaxy sustains a large population of black holes of mass around $10 M_{\odot}$ in low-mass binaries (more than $10^{3}$ ) or in isolation (more than $10^{7}$ ).

The black-hole candidates, with their nova-like extreme variability in X-rays (and in the optical), may point to the answer: the central massive black hole in the Galactic Centre is dormant, with mass not now accreting onto the central hole but gathering in a surrounding accretion disk for some future outburst. The X-ray novae seem to have duty cycles of luminous accretion of only about a month every 50 years or so and may be governed by an accretion limit-cycle instability in their accretion disk ${ }^{10}$. During their 'off' states, their accretion rate (and thus X-ray luminosity) is also about $10^{-7}$ of their Eddington value. If accretion onto the black hole in Sgr $A^{*}$ had a similar instability mechanism we should hardly expect to see it.

Thus although the rare class of galaxies with active nuclei may point to supermassive black holes now undergoing accretion, normal galaxies like the Milky Way or Andromeda (M31) may also harbour central massive black holes (although with masses lower by factors of $\left.10-10^{3}\right)$. The real difference is not the mass, or the mere presence; rather, it is the phase of the duty cycle. The active galactic nuclei are simply in the relatively rare 'on' state, while the normal galactic nuclei, such as Sgr $A^{*}$, are dormant like the vast majority of their brethren.

Jonathan E. Grindlay is at the HarvardSmithsonian Center for Astrophysics, 60 Garden Street, Cambridge, Massachusetts 02138, USA.

1. Goldwurm, A. et al. Nature 371, 589-591 (1994).

2. Bolton, C. T. Nature 235, 271-273(1972)

3. Van Paradijs, J. \& McClintock, J. E. in X-ray Binaries (eds Lewin, W. H. G., van Paradijs, J. \& van den Heuvel, E.P. J.) (Cambridge Univ. Press, in the press).

4. Sunyaev, R. et al. Astr. Astrophys. 247, L29-L32 (1991)

4. Zunyaev, R. et al. Astr. Astrophys. 247, L29-L32 (1991) 158, 811 (1964)

6. Rees, M. J. Observatory $98,210-232$ (1978)

7. Ford, H. et al. Astrophys. J. Lett. (in the press)

8. Genzel, R., Hollenbach, D. \& Townes, C. Rep. Prog. Phys. (in the press).

9. Grindlay, J. E., Manandhar, R. \& Covault, C. Astr. Astrophys. Suppl. 97, 155-158(1993).

10. Minishige, S., Kim, S. \& Wheeler, J. C. Astrophys. J. Lett. 358, L5-L8 (1990)

\section{Instant Newzak}

RADIO news has many advantages over the television variety. It is not burdened with compulsory pointless pictures; it doesn't seem obliged to make its newsreaders into celebrities; and you can catch up with it while doing other things. It could be brief, efficient and matter-of-fact. There was a blessed time when $B B C$ radio could straightforwardly announce, "There is no news tonight." And yet nowadays radio news expands to fill the space available for it - whole channels of desperate waffle in some cases. Daedalus wants to prune it back to its original efficiency.

In the studio, news exists as a number of stories in a 'stack', whose order depends on their newness and perceived importance. Any item slides down the stack with time; it features ever later and more briefly in successive newscasts, until it falls off the stack entirely. The simplest way of getting the stack to its consumers would simply be to broadcast it as a succession of updates, and accumulate it at the receiver for playback at any time.

The technology looks very simple. A sparse traffic of audio updates would not need a channel of its own; it could be coded and spread out to ride in the spaces of another radio channel, as Teletext does on a television transmission. At the receiver, it would be decoded and held in a memory chip of the type used in voice-mail and some audio processors. The receiver would update the news stack continuously as a 'background activity' - even when nominally switched off. The listener could hear the current stack at any time merely by touching a button. Compared with the money now being spent to realize the imagined need for video-ondemand transmissions, this news-ondemand system would be cheap indeed.

Furthermore, the listener could program and customize it to suit himself. A subject code attached to each item could be read by a programmable filter on the receiver itself. The listener could set the program to play back just those items he cared about (sport, politics, or whatever) and ignore the rest. News fanciers could stay abreast of their various fields of interest without having to sit through hours of irrelevant waffle. Television news (whose useful information is nearly all carried by its sound anyway) might lose some of its dangerous hypnotic power; millions of viewing hours could be saved for reality. And those of us who attend to the news purely negatively, to reassure ourselves that nothing important has happened, could gain that reassurance on demand, at any time.

David Jones 